\title{
Modeling stretched solitary waves along magnetic field lines
}

\author{
L. Muschietti, I. Roth, C. W. Carlson, and M. Berthomier \\ Space Sciences Laboratory, University of California, Berkeley, USA
}

Received: 25 June 2001 - Accepted: 16 October 2001

\begin{abstract}
A model is presented for a new type of fast solitary waves which is observed in downward current regions of the auroral zone. The three-dimensional, coherent structures are electrostatic, have a positive potential, and move along the magnetic field lines with speeds on the order of the electron drift. Their parallel potential profile is flattened and cannot fit to the Gaussian shape used in previous work. We develop a detailed BGK model which includes a flattened potential and an assumed cylindrical symmetry around a centric magnetic field line. The model envisions concentric shells of trapped electrons slowly drifting azimuthally while bouncing back and forth in the parallel direction. The electron dynamics is analysed in terms of three basic motions that occur on different time scales characterized by the cyclotron frequency $\Omega_{e}$, the bounce frequency $\omega_{b}$, and the azimuthal drift frequency $\omega_{\gamma}$. The ordering $\Omega_{e} \gg \omega_{b} \gg \omega_{\gamma}$ is required. Self-consistent distribution functions are calculated in terms of approximate constants of motion. Constraints on the parameters characterizing the amplitude and shape of the stretched solitary wave are discussed.
\end{abstract}

\section{Introduction}

One of the interesting findings of the FAST mission has been the presence of rapidly-moving solitary potentials in downward current regions of the auroral zone. Propagating along the magnetic field lines with a speed on the order of the electron drift, they appear as positive potential pulses of the order of hundreds of volts and create a bipolar electric signal: first directed upward, then downward. The structures were dubbed "fast solitary waves" to stress their large velocity ( $\sim 1000 \mathrm{~km} / \mathrm{s}$ ) (Ergun et al., 1998) and have been interpreted as traveling electron holes carried by the drifting electron distribution (Muschietti et al., 1999a). They may result from a two-stream instability occuring at lower altitudes (Goldman

Correspondence to: L. Muschietti

(laurent@ ssl.berkeley.edu) et al., 1999; Mandrake et al., 2000). Similar measurements have been reported by other satellites in the polar cap and the plasma sheet boundary layer (e.g. Tsurutani et al., 1998; Cattell et al., 1999; Franz et al., 2000).

Earlier investigations (Ergun et al., 1998, 1999) of these fast solitary waves focused on structures for which the electric signal in the parallel direction fits well to the derivative of a Gaussian. Among the potential structures observed by FAST, however, there is another class of intense structures where the conjugate electric spikes are set apart (see Fig. 1 bottom) and thus cannot fit to the derivative of a Gaussian. These dispersed bipolar spikes have also been identified by Tsurutani et al. (1998) in the polar cap data set of the Polar satellite. The underlying potential structure, whose passage by the satellite creates the bipolar electric signal, must be somewhat boxy with a potential profile much flatter than a Gaussian. In this article we present an extended BGK (Bernstein et al., 1957) model for these structures, that we call "stretched solitary waves".

The bipolar signal observed in the parallel direction is accompanied by a unipolar electric signal in the perpendicular direction, showing that the structure is at least two or three-dimensional. We improve upon our previous theoretical modeling (Muschietti et al., 1999a) by including a perpendicular profile for the potential. While an experimental determination of the perpendicular profile is impossible due to the parallel motion of the structure relative to the spacecraft, an adequate description of the perpendicular electric field is desirable. The latter is likely responsible for the enhanced ion heating found during periods of intense solitary waves as evidenced by the $90^{\circ}$ conics (Ergun et al., 1999). The potential model presented here has a radial dependence with an assumed cylindrical symmetry, and as such can describe the unipolar electric field recorded in the perpendicular direction.

The plasma environment of the spikes observed by FAST is strongly magnetized with a ratio gyro-to-plasma frequency $\Omega_{e} / \omega_{e}>5$, a large parallel Debye length of the order of $100 \mathrm{~m}$, and a small electron gyroradius less than a meter. 

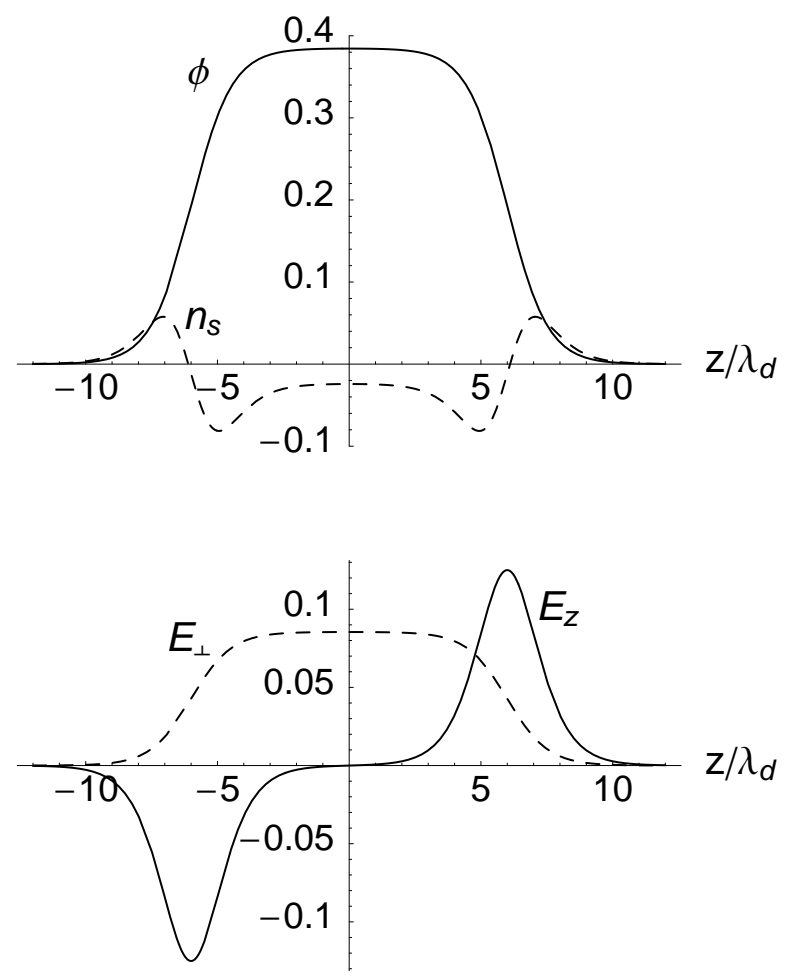

Fig. 1. Example of stretched solitary wave showing the profiles of various quantities along $z$, distance parallel to the magnetic field $\boldsymbol{B}$. (Top) Profiles of the potential $\phi$ and the associated density $n_{S}$ (dashed curve). (Bottom) Parallel and perpendicular profiles of the electric field. The plot is computed from Eqs. (1), (2), (3), and (4) with the parameters: $\psi=0.6, \Delta=6, \beta=1.3$, and $\delta_{\perp}=6$. Distance off the centric magnetic field line $r=4$. Note the two conjugate electric spikes $E_{z}$ separated by a distance $2 \Delta$.

With electrons tightly tied to a magnetic field line, their dynamics is characterized by motions on different time scales of which the cyclotron period is by far the shortest. This enables us to build a three-dimensional trapped distribution consistent with both parallel and perpendicular fields.

In Sect. 2 the model of potential is presented as well as the density perturbation it requires. The orbits of electrons in the presence of such a potential are examined in Sect. 3 . Using the BGK method, we build a trapped distribution consistent with the potential model in Sect. 4. Finally, we close the paper with a short discussion in Sect. 5. Throughout the article one uses the standard dimensionless units where length is normalized by the Debye length $\lambda_{d}$, time by the inverse plasma frequency $\omega_{e}^{-1}$, velocity by the (parallel) electron thermal velocity $v_{e \|} \equiv \sqrt{T_{e} / m}=\omega_{e} \lambda_{d}$, and potential expressed in $m v_{e \|}^{2} / e$ units.

\section{Potential model}

We assume that there exists a frame moving along $\boldsymbol{B}$ in which potential and electron distribution are in self-consistent steady state. Working in this frame, a potential cylindrically

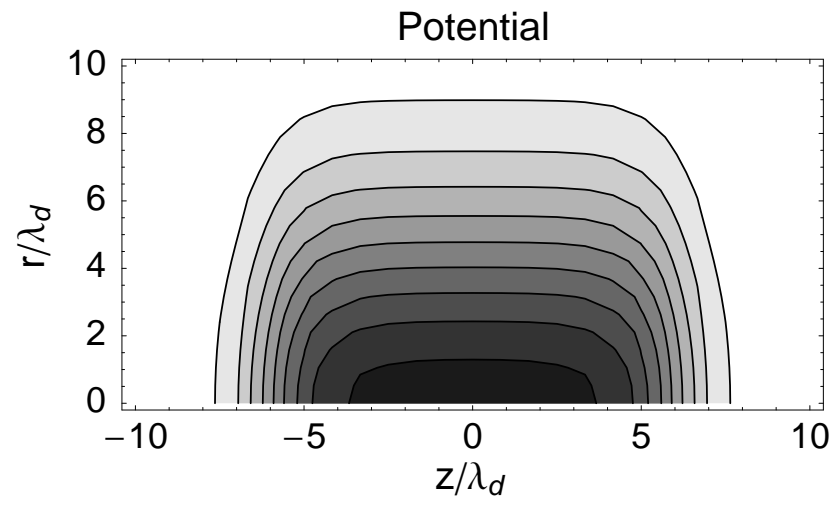

Fig. 2. A contour plot representation of the potential model. The parameter values are as for Fig. 1. The 9 contours are linearly spaced from 0.56 down to 0.06 in units of $m v_{e \|}^{2} / e$. Note the stretching along $z$.

symmetric around a centric magnetic field line is defined in terms of $z$, the coordinate along $\boldsymbol{B}$, and $r=\sqrt{x^{2}+y^{2}}$, the radius away from the centric line:

$\phi(z, r)=\psi h(z) a(r)$

with $h(z)=[1+\eta \cosh (\beta z)]^{-1}$

and $a(r)=\exp \left[-\left(r / \delta_{\perp}\right)^{2}\right]$.

An illustration of the profile along $z$ is shown in Fig. 1 . The parameter $\beta$ determines the width of the electric spikes, or alternately the parallel gradients of the potential where these are strong. The two spikes are separated by the distance $2 \Delta$, which can be several Debye lengths. This is also the length of the perpendicular electric pulse. The derived parameter $\eta \equiv 2 \exp (-\beta \Delta)$ is normally a small number, typically less than 0.01 , whereby the potential profile is flat about the top. Only where $|z|>-(1 / \beta) \ln (2 \eta)$, does the potential significantly drop from its maximum, $\psi /(1+\eta) \simeq \psi$. For $\eta \rightarrow 1 / 2$, one recovers a peaked potential similar to the case we previously studied (Muschietti et al., 1999b). As for the radial dependence $a(r)$, we choose a Gaussian fall-off with characteristic width $\delta_{\perp}$. The choice is arbitrary and dictated for the sake of simplicity only. A view of the potential by means of contours is shown in Fig. 2.

The parallel electric field $E_{z}$, the radial electric field $E_{\perp}$, and the density perturbation $n_{s}$ are obtained through derivations

$$
\begin{aligned}
& E_{z}(z, r)=\beta \psi a(r) \frac{\eta \sinh (\beta z)}{[1+\eta \cosh (\beta z)]^{2}} \\
& E_{\perp}(z, r)=\frac{\psi a(r)}{1+\eta \cosh (\beta z)} \frac{2 r}{\delta_{\perp}^{2}} .
\end{aligned}
$$

A display of the profiles of $E_{z}$ and $E_{\perp}$ as a function of $z$ is visible in Fig. 1. Due to the relative motion between spacecraft and potential structure, the latter is sampled along $\boldsymbol{B}$. Thus, the profiles in Fig. 1 represent the idealized electric signal to be detected, according to the model, for a given 


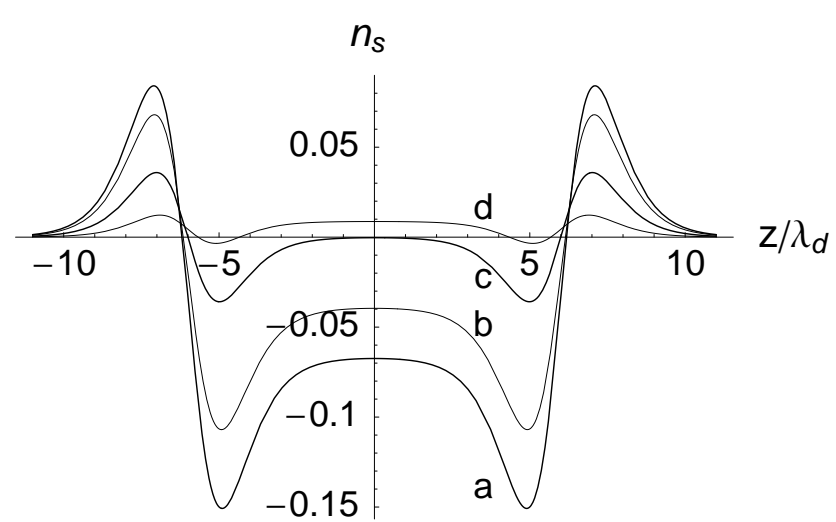

Fig. 3. Profiles of the density $n_{s}$ along $z$ at various radii: (a) $r=0$, (b) $r=\delta_{\perp} / 2$, (c) $r=\delta_{\perp}$, (d) $r=3 \delta_{\perp} / 2$. Note the similitude between the different radii. There are differences though in the relative weight between density enhancements at $|z|>\Delta$ and density perturbations about $z \sim 0$ due to the $r$-dependence in the last term of Eq. (4). The inhomogeneous density is for the most part due to the electrons trapped in the potential. Parameters as in Fig. 1: $\psi=0.6, \Delta=6, \beta=1.3$, and $\delta_{\perp}=6$.

"impact parameter" off the centric magnetic field line. At $r$ fixed (here $r=4$ ), the peak amplitude of the bipolar spikes is $(\beta \psi / 4) a(r)$, while that of the unipolar signal in the perpendicular direction is $2 \psi r a(r) / \delta_{\perp}^{2}$. The electron density perturbation is given by Poisson equation as

$$
\begin{aligned}
n_{s}(z, r) & =\beta^{2} \frac{\eta \psi a(r)}{2[1+\eta \cosh (\beta z)]^{3}} \\
& \times\left[-2 \eta-\cosh (\beta z)+\eta \cosh ^{2}(\beta z)\right] \\
& +\frac{2 \psi a(r)}{1+\eta \cosh (\beta z)} \frac{r^{2}-\delta_{\perp}^{2}}{\delta_{\perp}^{4}}
\end{aligned}
$$

The case of a planar structure is recovered with $\delta_{\perp} \rightarrow \infty$, hence $a(r) \rightarrow 1$. Then, the radial field $E_{\perp}$ vanishes and the density perturbation $n_{s}$ (4) reduces to a simpler expression without the last term in $\delta_{\perp}^{-4}$. The effect of this term is to decrease the overall density at small radius and increase it for $r>\delta_{\perp}$. Figure 3 shows the perturbed density profile as a function of the distance along $\boldsymbol{B}$ for different radii. The perturbation is symmetric in $z$ and includes an enhancement for $|z|>\Delta$, a depletion for $|z|<\Delta$, and a more neutral region around $z \sim 0$. To understand how an electron population can behave in order to self-consistently maintain such a perturbation, we need to examine the electron orbits.

\section{Electron dynamics}

Electrons in the presence of potential (1) obey the following equations of motion:

$\ddot{x}+\Omega_{e} \dot{y}=-\psi h(z) a\left(\sqrt{x^{2}+y^{2}}\right) \frac{2 x}{\delta_{\perp}^{2}}$
$\ddot{y}-\Omega_{e} \dot{x}=-\psi h(z) a\left(\sqrt{x^{2}+y^{2}}\right) \frac{2 y}{\delta_{\perp}^{2}}$

$\ddot{z}=-E_{z}\left(z, \sqrt{x^{2}+y^{2}}\right)$,

where $\Omega_{e}$ is the electron cyclotron frequency in absolute value. We have numerically integrated the equations, which showed that the electron behaviour can be analysed in terms of three basic motions occuring on different time scales: the fast cyclotron gyration, the parallel motion including the bounce back and forth of the trapped particles, and a slow azimuthal drift along a shell at constant radius. An important consideration is the parallel distance covered by the electrons during a cyclotron period. If the distance is short compared to the parallel scale length of $\phi$, the term $h(z)$ in Eqs. (5) and (6) can be considered constant for the time of a gyration. While this condition is not satisfied for the fast passing particles, it is satisfied for those electrons whose dynamics is the most important to support the potential structure, namely the trapped ones and the slow passing ones that are close to the separatrix. These are strongly accelerated then decelerated, hence they contribute significantly to the density perturbation which has to self-consistently create the potential. Since their parallel velocity is less than $\max \dot{z} \sim \sqrt{\psi}$, the condition can be expressed as $\beta \sqrt{\psi} \ll \Omega_{e}$. It is reminiscent of the relation between the bounce frequency $\omega_{b}$ and $\Omega_{e}$, which is necessary for the existence of electron phase-space holes in a magnetized plasma (Muschietti et al., 2000). The bounce period associated with the stretched solitary waves is discussed below.

Since the cyclotron gyration in the $[x, y]$ plane is the fastest eigenmotion, we rewrite Eqs. (5) and (6) in terms of the independent time variable $\tau=\Omega_{e} t$. This yields the set

$\ddot{x}+\dot{y}=-\epsilon h(z) a\left(\sqrt{x^{2}+y^{2}}\right) 2 x$

$\ddot{y}-\dot{x}=-\epsilon h(z) a\left(\sqrt{x^{2}+y^{2}}\right) 2 y$,

which features the small parameter $\epsilon \equiv \psi /\left(\Omega_{e} \delta_{\perp}\right)^{2} \ll 1$. To solve the set of equations we apply the two time scale method (e.g. Bender and Orszag , 1978, p. 549) where the slow time is $\tilde{\tau}=\epsilon \tau$. Gyrocenter coordinates are introduced through the ansatz

$x=R_{x}(\tilde{\tau})+\rho \cos (\tau+\theta(\tilde{\tau}))$

$y=R_{y}(\tilde{\tau})+\rho \sin (\tau+\theta(\tilde{\tau}))$,

where $\theta$ is the gyrophase and $R_{x}, R_{y}$ denote the gyrocenter's position. We assume that the gyroradius is constant and furthermore, that the radial profile $a(r)$ varies little over a gyroradius, a very reasonable assumption in FAST's environment. After substitution of the ansatz and an expansion to terms of the order of $O\left(\epsilon^{2}\right)$, one obtains these dynamical equations for the slow evolution,

$\frac{d R_{x}}{d \tilde{\tau}}=+2 h(z) a(R) R_{y}$
$\frac{d R_{y}}{d \tilde{\tau}}=-2 h(z) a(R) R_{x}$ 
$\frac{d \theta}{d \tilde{\tau}}=2 h(z) a(R)$,

with $R \equiv \sqrt{R_{x}^{2}+R_{y}^{2}}$ and where terms of the order of $\left(\rho / \delta_{\perp}\right)^{2}$ have been neglected. The two Eqs. (10) and (11) describe an azimuthal drift of the gyrocenter on a shell of constant radius and Eq. (12) describes a frequency shift. Thus, the quantity $R$ is a constant of motion. Let a time-dependent angle $\gamma(t)$ denote the azimuth of the electron gyrocenter: $R_{x}=R \cos (\gamma), R_{y}=R \sin (\gamma)$. Returning to the original unit of time, we obtain from either Eqs. (10) or (11) an equation for the slow azimuthal drift

$\dot{\gamma}=-\frac{2 \psi}{\Omega_{e} \delta_{\perp}^{2}} h(z) a(R)$.

Equation (12) shows that in association to the drift, the gyration frequency is slightly altered from $\Omega_{e}$ to $\Omega_{e}-\dot{\gamma}$. Note that because $z=z(t)$ does change on the time scale of the drift, the quantity $h(z)$ varies, hence the rate $\dot{\gamma}$ is not constant.

\subsection{Passing electrons}

Figure 4 shows the parallel velocity $v_{z}$ and the perpendicular velocity $v_{\perp}$ for an electron that crosses the potential structure slightly off the centric magnetic line. The particle approaches slowly the positive potential, its parallel velocity strongly increases on the upslope then decreases on the downslope. Meanwhile, the perpendicular velocity is constant, oscillates as the particle crosses the potential structure, and regains nearly the same constant value on the downstream side. The oscillations are due to the radial electric field $E_{\perp}$. Using the gyrocenter coordinates, we can rewrite the perpendicular (by contrast to parallel) velocity $v_{\perp} \equiv\left(\dot{x}^{2}+\dot{y}^{2}\right)^{1 / 2}$ as

$$
\begin{aligned}
& v_{\perp}(t)=\left[\rho^{2}\left(\Omega_{e}-\dot{\gamma}\right)^{2}+R^{2} \dot{\gamma}^{2}\right. \\
& \left.\quad+2 R \rho\left(\Omega_{e}-\dot{\gamma}\right) \dot{\gamma} \cos \left(\Omega_{e} t-2 \gamma(t)+\gamma_{0}\right)\right]^{\frac{1}{2}}
\end{aligned}
$$

with $\gamma_{0}$ the azimuth of the gyrocenter at $t=0$. The velocity oscillates at approximately the cyclotron frequency between $\left|\rho \Omega_{e} \pm(R \mp \rho) \dot{\gamma}\right|$, while the electron crosses the potential structure, meaning $\dot{\gamma} \neq 0$.

This behaviour enables us to average out the oscillations in the perpendicular kinetic energy. As a result, the parallel energy

$w \equiv v_{z}^{2}-2 \phi(z, R)$

is a constant of motion on the slower time scale. We assume a distribution of passing electrons of the form $f_{e}(w)$, which is homogeneous away from the potential structure yet becomes radially inhomogeneous near the structure. The density of passing electrons $n_{p}$ decreases in the vicinity of the potential structure as they are accelerated by the positive potential. The actual amount of decrease depends upon their distribution as is clear from the density integral

$n_{p}(\phi)=\int_{0}^{\infty} \frac{1}{2}\left[f_{e}^{+}(w)+f_{e}^{-}(w)\right](w+2 \phi)^{-1 / 2} d w$, where $f_{e}^{+}, f_{e}^{-}$refer to right-moving and left-moving electrons, respectively.

The electron distributions measured by FAST are drifting and broad in the parallel direction with a flat-top maximum (Carlson et al., 1998). In the satellite frame, the electron distributions appear to have an average drift smaller or comparable to the velocity of the spikes (Ergun et al., 1999). We shall here assume that the electron drift is zero in the frame of the potential structure. For simplicity, we shall furthermore assume that the distributions are symmetric, $f_{e}^{+}(w)=f_{e}^{-}(w)=f_{e}(w)$, and use the same model as in Muschietti et al. (1999a):

$f_{e}(w)=\frac{6 \sqrt{2}}{\pi\left(8+w^{3}\right)} \quad$ with $w>0$.

The "temperature", or spread, $2 \int_{0}^{\infty} d v v^{2} f_{e}\left(v^{2}\right)$, is normalized to unity. An electron with $w=v^{2}=1$ is a "thermal" electron (in the solitary structure's frame). For smaller energies, $f_{e}(w)$ is flat, while it drops as a power-law for larger energies.

Although there is no simple explicit expression for $n_{p}(\phi)$ that can be obtained with the distribution (17), one can use an expansion for small $\phi$ to analyse the behaviour of the passing electrons (Krasovsky et al., 1997),

$n_{p}(\phi)=1-\frac{3 \phi^{1 / 2}}{\pi}+\frac{\phi}{2}-\frac{3 \phi^{2}}{16}+O\left(\phi^{5 / 2}\right)$.

The main response is a reduction in density scaling as $\phi^{1 / 2}$. An obvious yet important consequence is that the density of passing electrons is depleted and approximately constant between $-\Delta+\beta^{-1}<z<+\Delta-\beta^{-1}$. Therefore, it is the role of the trapped electrons to provide the structured density profile seen in Fig. 3.

\subsection{Trapped electrons}

As the particles move back and forth in the parallel direction, their gyrocenters remain on the same shell at constant $R$. Due to the radial electric field, these gyrocenters drift in the azimuthal direction. Figure 5 illustrates the motion of one trapped electron by showing two phase-space views. The orbits were obtained from numerically integrating Eqs. (5), (6), and (7). The left plot depicts the slow azimuthal drift at constant radius and displays a trochoid-like orbit. The right plot depicts the variation of the two components $v_{x}$ and $v_{y}$ of the perpendicular velocity; the latter rapidly oscillates between a minimum and maximum value, as described by Eq. (14).

Since $z=z(t)$, the azimuthal drift $\dot{\gamma}$ (see Eq. 13) is, in general, not constant. However, for many trapped electrons the bounce period is short as compared to the drift time, so that one can average $h(z)$ over the periodic bounce motion. Such is the case for the electron orbit shown in Fig. 5. This orbit has a bounce frequency $\omega_{b}=0.1 \Omega_{e}$ and a turning point at $|z|=2.2$, less than the potential width $\Delta=4$. Hence, assuming $\psi h(z) \approx \psi=0.4$ and substituting the numerical values of $R=3, \delta_{\perp}=6$, one finds $\dot{\gamma}=0.017 \Omega_{e}^{-1}$. In 

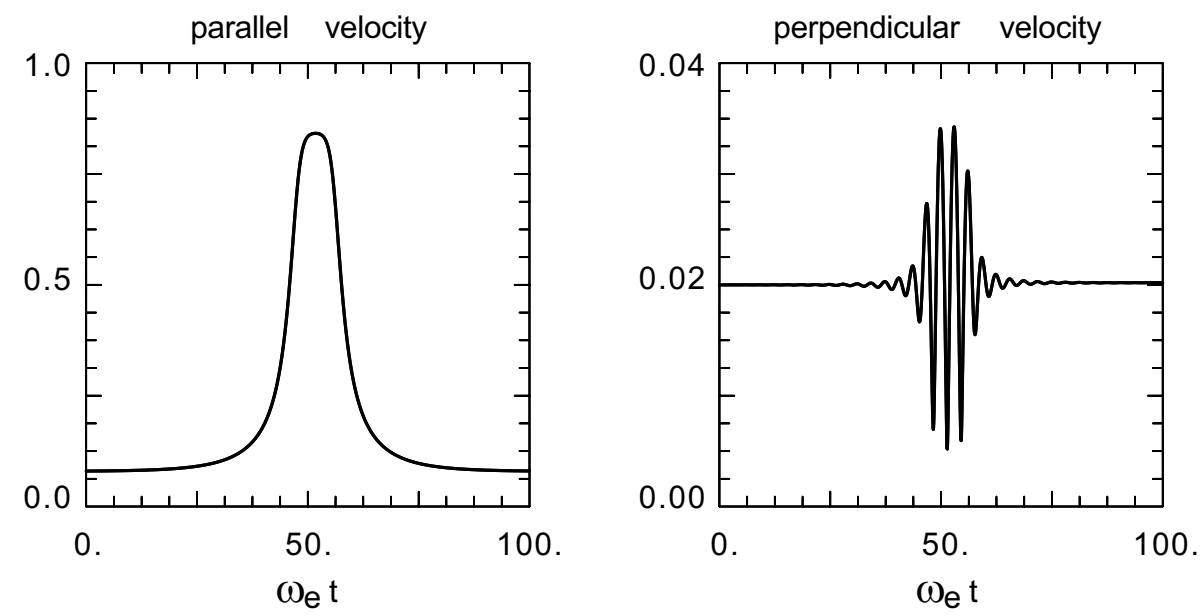

Fig. 4. Evolution of the parallel and perpendicular velocity for a slowly passing electron. Note the acceleration/deceleration in $v_{\|}$and the oscillations in $v_{\perp} \equiv\left(v_{x}^{2}+v_{y}^{2}\right)^{1 / 2}$ as the particle crosses the potential structure about $\omega_{e} t=50$. The oscillations are caused by the radial electric field, occur close to the frequency $\Omega_{e}$, and average to nearly zero change in perpendicular kinetic energy (see Eq. 14). Parameters: $\psi=0.4, \Delta=4, \beta=1.5$, and $\delta_{\perp}=6$.
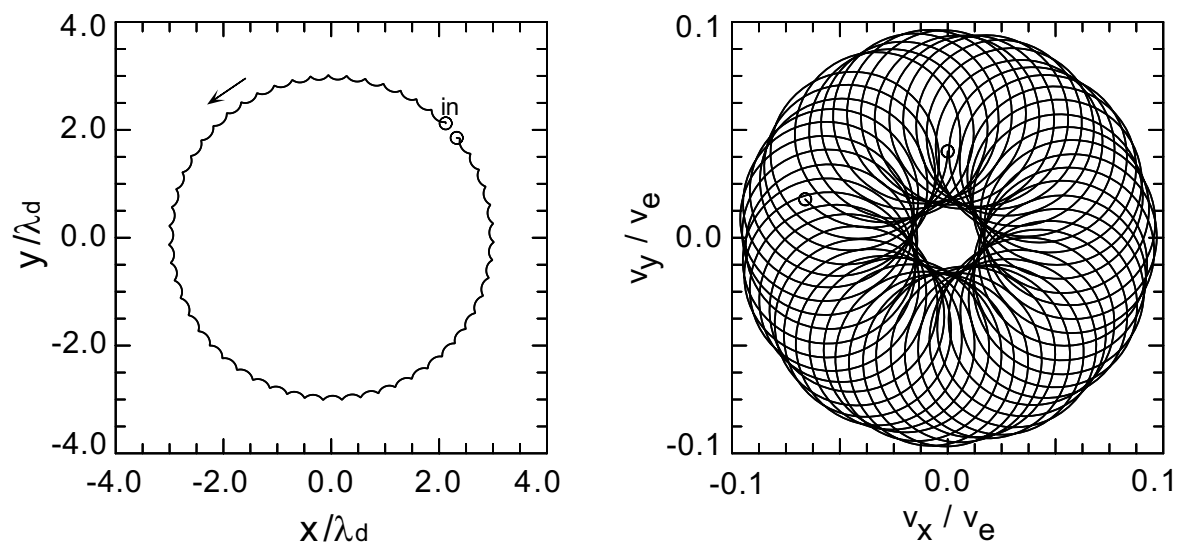

Fig. 5. Orbit of a trapped electron projected in the perpendicular $(x, y)$ plane for a magnetic field $\boldsymbol{B}$ pointing in the $-\hat{z}$ direction. (Left) $(x, y)$ coordinates describe an azimuthal drift with a trochoid-like orbit. "In" refers to initial position; See text near Eq. (13) for details. (Right) $v_{x}$ and $v_{y}$ oscillate at frequency $\Omega_{e}$ around an averaged $v_{\perp}$ value. Parameters: $\psi=0.4, \Delta=4, \beta=1.5$, and $\delta_{\perp}=6$. The additional choice $\Omega_{e}=\omega_{e}$ brings cyclotron and azimuthal time scales closer for the sake of plot legibility.

dimensional units this reads $\dot{\gamma} / \Omega_{e}=0.017\left(\omega_{e} / \Omega_{e}\right)^{2}$ and shows that the azimuthal time scale is indeed very long as compared to the bounce period and the cyclotron period. The three time scales follow the ordering

$\dot{\gamma} \ll \omega_{b} \ll \Omega_{e}$.

The term $h(z)$ can thus be replaced by a value averaged over the trapped orbit (denoted by \langle\rangle ), and the angular drift becomes a constant $\omega_{\gamma}$ :

$\omega_{\gamma}=-\frac{2 \psi}{\Omega_{e} \delta_{\perp}^{2}}\langle h\rangle a(R)$.

The constant angular drift is visible in Fig. 5. In the left panel, it shows as the regular indentation of the trochoid-like orbit. The magnetic field $\boldsymbol{B}$ was set to point in the $-\hat{z}$ direction, hence the positive angular drift. In the right panel, the perpendicular velocity is seen to regularly oscillate between a minimum and maximum value. Upon replacing $\dot{\gamma}$ in Eq. (14) by $\omega_{\gamma}$, one obtains exactly this behaviour with the perpendicular velocity oscillating at the frequency $\Omega_{e}-2 \omega_{\gamma} \approx \Omega_{e}$ between

$$
\begin{aligned}
\min \left[v_{\perp}^{2}\right] & =\left[\omega_{\gamma}(R+\rho)-\Omega_{e} \rho\right]^{2} \quad \text { and } \\
\max \left[v_{\perp}^{2}\right] & =\left[\omega_{\gamma}(R-\rho)+\Omega_{e} \rho\right]^{2} .
\end{aligned}
$$

On average, the perpendicular energy is a constant equal to $\omega_{\gamma}^{2} R^{2}+\left(\Omega_{e}-\omega_{\gamma}\right)^{2} \rho^{2}$. Therefore, $w$ defined in Eq. (15) as the parallel energy is also a constant.

The bounce period depends upon the particle's parallel energy $w$. For the potential of a stretched solitary wave, this dependence is peculiar, decreasing at first for deeply trapped electrons (see Fig. 6). This characteristic comes from the very shallow potential well at low energies. From Eq. (15), 
we obtain a formal expression for the period of an oscillatory motion between symmetric turning points $\pm_{\tau}(w)$ :

$T_{b}=\frac{4}{[2 \psi a+w]^{\frac{1}{2}}} \int_{0}^{z_{\tau}(w)} d z\left[\frac{1+\eta \cosh (\beta z)}{1+\frac{\eta w}{2 \psi a+w} \cosh (\beta z)}\right]^{\frac{1}{2}}$,

where $a=a(R)$ has a fixed value associated with the shell where the electron gyrocenter lies. Kinetic energy and turning point are related via $2 \psi a+w=-\eta w \cosh \left(\beta z_{\tau}\right)$. If the kinetic energy is not too small, $2 \psi a+w \gg-\eta w$, we can approximate the integrand by

$$
\left(1+e^{\beta(z-\Delta)}\right)^{\frac{1}{2}}\left(1+\frac{w}{2 \psi a+w} e^{\beta(z-\Delta)}\right)^{-\frac{1}{2}}
$$

$[\mathrm{t}]$

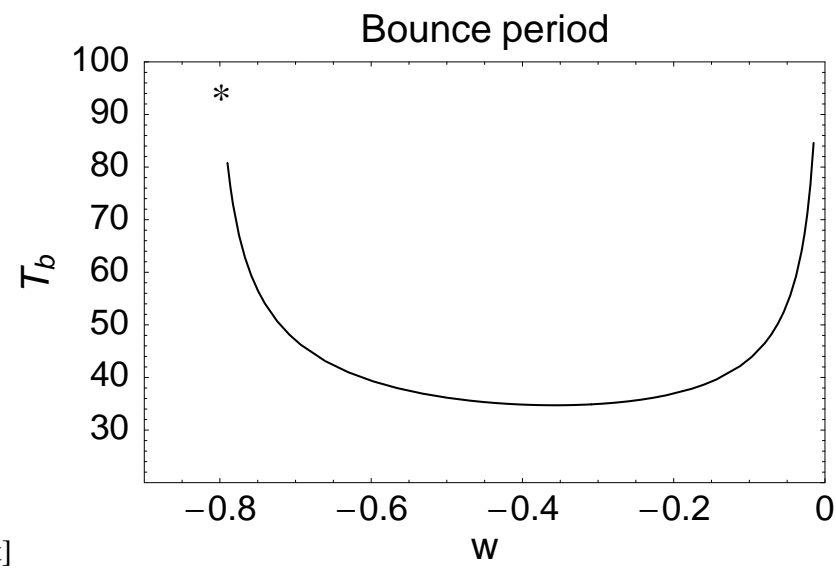

Fig. 6. Bounce period for an electron trapped in a stretched solitary wave. Shown dependence on energy $w$ is from Eq. (22). The very shallow potential well leads to the peculiar behaviour for lower energies, whereby $T_{b}$ increases toward $T_{b 0}$ at the well's bottom (* given by Eq. 23). Parameters: $\psi=0.4, \Delta=6, \beta=1.5$, and $r=0$.

$$
+\frac{4}{\beta \sqrt{-w}}\left[\frac{\pi}{2}+\arctan \left(\frac{w+\psi a}{\sqrt{-w(2 \psi a+w)}}\right)\right] .
$$

With $w$ increasing, the term on the first line decreases, whereas the term on the second line increases. At large energies the second term dominates, which is independent from $\Delta$, the parallel size of the potential structure. At small energies the first term dominates, which is proportional to $\Delta$. If the kinetic energy is vanishingly small, Eq. (22) breaks down; then the period is given by

$T_{b 0}=\frac{2 \pi}{\beta \sqrt{\eta \psi a}}$,

which contains $\Delta$ through the factor $\eta \equiv 2 e^{-\beta \Delta}$. The solid curve in Fig. 6 shows Eq. (22) as a function of $w$ for $\psi=0.4$, $\Delta=4, \beta=1.5$, and $R=0$, while the star indicates the value at the bottom of the potential well (Eq. 23).

The analysis above demonstrates that the condition $\omega_{\gamma} \ll \omega_{b}$ can be satisfied for a large class of trapped particles. Also, to require a sufficiently long drift period by combining Eqs. (20) and (23) yields a bound on the minimum perpendicular size for the solitary wave

$\delta_{\perp}^{2} \gg \frac{2}{\Omega_{e} \beta} \sqrt{\frac{\psi}{\eta}}$.

The weaker the magnetic field, the larger the perpendicular scale needs to be. Note also that a larger parallel size $(\eta \rightarrow 0)$ imposes a larger perpendicular scale. Implications for the space observations are discussed below in Sect. 5 .

\section{Trapped distribution function}

The net density perturbation $n_{s}$ required by the shape of our cylindrically symmetric potential is made up of both the trapped electron density $n_{t}$ and the response of the passing electrons $n_{p}: n_{s}=n_{t}+n_{p}-1$. Here, -1 represents the ions which we assume to form an homogeneous, neutralizing background. Considering the results of Sect. 3, we write the density of trapped electrons on each shell $R$ as

$n_{t}(\phi ; R)=\int_{-2 \phi}^{0} \frac{f_{t}(w ; R)}{(w+2 \phi)^{\frac{1}{2}}} d w$,

where $f_{t}(w ; R)$ denotes the trapped distribution and $R$ is considered from now on a parameter. Our goal is to find an explicit expression for $f_{t}(w ; R)$. The integral Eq. (25) can be inverted for $f_{t}(w ; R)$ with the help of Laplace transforms (see Sect. 2.3 in Muschietti et al. (1999b) for details). The result reads

$f_{t}(w ; R)=\frac{1}{2 \pi} \int_{0}^{-w}(-w-p)^{-\frac{1}{2}} \frac{d}{d p} n_{t}(p ; R) d p$,

where the range of possible energies $w$ differs on each shell,

$-2 \psi a(R) \leq w<0$.

Now since $n_{t}(\phi ; R)$ is made up of $n_{s}(\phi ; R)$ and $n_{p}(\phi)$, the quadrature Eq. (26) splits into two: one for $n_{s}$ and one for $n_{p}$. Using the relation between the value of the potential $\phi$ and the position $z$ for a given $R$

$1+\eta \cosh (\beta z)=\psi a(R) / \phi$,

one rewrites the density perturbation $n_{s}$ of Eq. (4) into

$$
\begin{aligned}
& n_{S}(\phi ; R)=\left(\frac{\beta}{\psi a(R)}\right)^{2} \phi^{2}\left[2\left(1-\eta^{2}\right) \phi-3 \psi a(R)\right] \\
& \quad+\phi\left[\beta^{2}-\frac{4}{\delta_{\perp}^{2}}\left(1-\frac{R^{2}}{\delta_{\perp}^{2}}\right)\right] .
\end{aligned}
$$



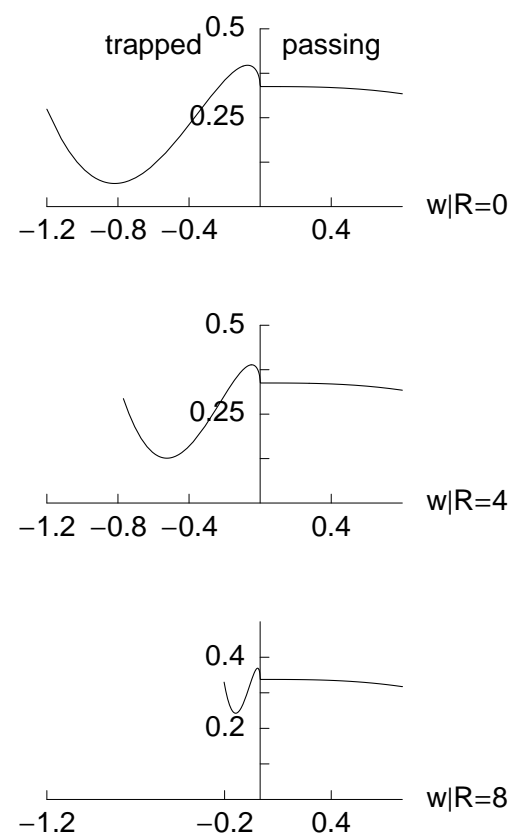

Fig. 7. Distribution as a function of the parallel energy $w$ for three radii $R$. The plot is computed from Eq. (34) with the same parameters as in Fig. 1: $\psi=0.6, \Delta=6, \beta=1.3$, and $\delta_{\perp}=6$. Note the enhancement of deeply trapped electrons ( $w$ near minimum) and the dearth of more energetic trapped electrons. As the location of the shell $R$ increases, the trapped part shrinks to negligible importance.

After differentiating Eq. (29), we substitute the expression into Eq. (26). The resulting quadrature can be integrated exactly and yields

$$
\begin{gathered}
f_{t s}(w ; R)=\frac{\beta^{2}}{\pi}(2 \psi a)^{\frac{1}{2}}\left\{\frac{16}{5}\left(1-\eta^{2}\right) u^{\frac{5}{2}}-4 u^{\frac{3}{2}}\right. \\
\left.+u^{\frac{1}{2}}\left[1-\frac{4}{\beta^{2} \delta_{\perp}^{2}}\left(1-\frac{R^{2}}{\delta_{\perp}^{2}}\right)\right]\right\}
\end{gathered}
$$

with $u \equiv-w /(2 \psi a)$, where the subscript $s$ associates the quadrature with $n_{s}$. Recall $\eta$ is normally very small, $\eta \ll 1$, and the perpendicular scale is expected to make $\beta \delta_{\perp} \gg 2$. The expression above is a non-monotonous function of $w$ through the three powers of $u$. Starting from the separatrix, $w=0$, with $w$ increasingly negative, $u$ varies from zero to one. Hence, $f_{t s}$ first rises from zero because of the $u^{1 / 2}$ term, then dips due to the $u^{3 / 2}$ term, to finally bounce back for deeply trapped electrons, $u \lesssim 1$. Assuming $\eta \ll 1$ and $\beta \delta_{\perp} \gg 2$, one easily can find the minimum of $f_{t s}(w ; R)$. It occurs for

$w_{0} /(2 \psi a)=-(3+\sqrt{5}) / 8$

where $f_{t s}$ reaches the negative value

$f_{t s}\left(w_{0} ; R\right)=-\beta^{2}(\psi a)^{\frac{1}{2}} \frac{\sqrt{5}-1}{10 \pi}(3+\sqrt{5})^{\frac{1}{2}}$.
The second quadrature, associated with $n_{p}$, is exactly that which we previously calculated since we use the same model of passing electrons (Muschietti et al., 1999b). It yields

$f_{t p}(w)=\frac{6+(\sqrt{2}+\sqrt{-w})(1-w) \sqrt{-w}}{\pi(\sqrt{2}+\sqrt{-w})\left(4-2 w+w^{2}\right)}$

which is monotonously and slowly decreasing as $w$ becomes increasingly negative. The trapped distribution function is obtained by adding the two quadratures Eqs. (30) and (33),

$f_{t}(w ; R)=f_{t s}(w ; R)+f_{t p}(w)$.

The behaviour of $f_{t}(w ; R)$ is illustrated in Fig. 7, which shows the distribution as a function of the parallel energy for 3 different radii. On the left, the trapped part is displayed over its range of possible energies $(-2 \psi a(R) \leq w<0)$; on the right $(w>0)$ just a short section of the passing distribution is shown. The trapped part of the distribution displays the minimum at $w_{0}$ from Eq. (31) and shrinks as the radius increases, a reflection of the "self-similarity" embedded in the variable $u$ of Eq. (30).

Figure 8 provides an illustration of the distribution function in the familiar $(z, v)$ phase-space. It shows

$F(z, v)=\left\{\begin{array}{ll}f_{t}\left(w=v^{2}-2 \phi(z)\right), & \text { if } w<0 \\ f_{e}\left(w=v^{2}-2 \phi(z)\right), & \text { if } w>0\end{array}\right.$,

where we have set $R=0$. Note the complex structure of the distribution, which exhibits a narrow lip near the separatrix and a secondary bulge about $z=0, v=0$ within the large "caldera". This enhanced trapped population is characteristic of the stretched solitary wave. Its role is to offset the decreased density of passing electrons in order to produce the weak curvature of the potential in the flat-top region. It does not exist for a "classic" Gaussian potential (Muschietti et al., 1999a), which has a substantial curvature at its center. Note also how the flanks of $F$ swell for $-6<z / \lambda_{d}<6$. This feature is expected to translate into a broadening of the electron distributions observed in the spacecraft frame for the time interval between the two conjugate parallel electric spikes.

\section{Discussion}

We have constructed a BGK nonlinear object that models a new type of fast solitary wave observed in the auroral zone. The positive potential has a parallel profile with a flat maximum between the opposite gradients, which sets apart the conjugate electric spikes. The parameter measuring the distance between the two spikes is $2 \Delta$, while that measuring their individual width is $\beta^{-1}$. The potential model includes a perpendicular Gaussian profile with an assumed cylindrical symmetry and a characteristic width $\delta_{\perp}$. As such, it predicts the detection of a unipolar perpendicular electric pulse that is delimited by the two parallel spikes (see Fig. 1), as observed. Cylindrical symmetry with concentric shells of electrons slowly drifting azimuthally, while bouncing back and 


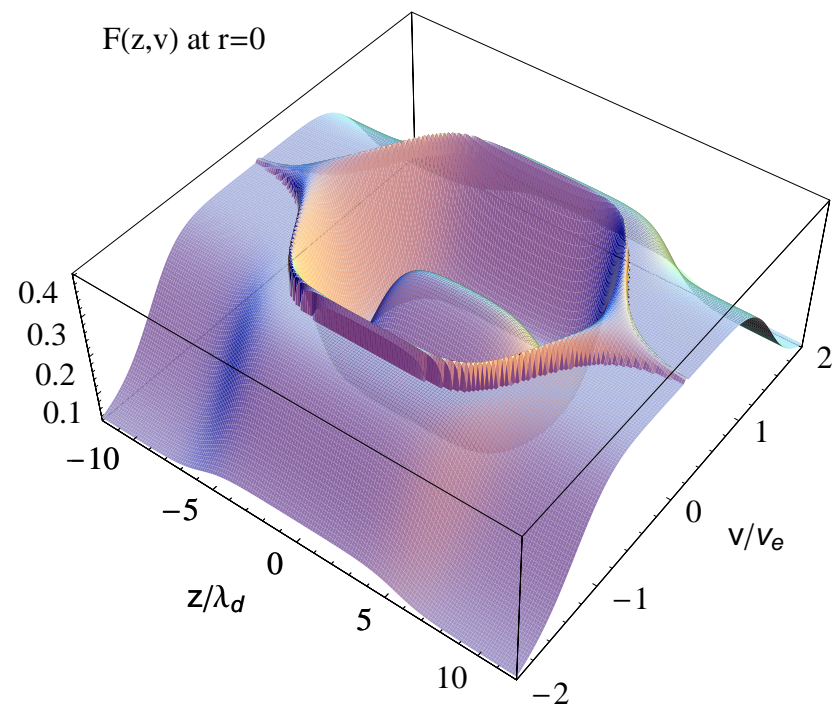

Fig. 8. Distribution function in $\left(z, v_{z}\right)$ space where $z$ and $v_{z}$ denote the position and velocity in the parallel direction. Perspective view made from Eq. (35) with same parameters as in Fig. 7 and $R=0$. Note the bulge about $z=0$ and $v=0$ within the large "caldera", a characteristic signature of the stretched solitary wave.

forth in the parallel direction, makes the potential structure self-consistent and contained in a finite portion of the threedimensional space. The construction of such an object imposes a number of constraints on the parameters $\Delta, \beta$, and $\delta_{\perp}$, the significance of which we examine now.

First, as in the case of the classic electron hole, there is a relation between the amplitude and the parallel scale length of $\phi$. The condition comes from imposing that $f_{t}(w ; R) \geq 0$ where it reaches minimum. Setting $R=0$ in Eqs. (31) and (32), one obtains

$\beta^{2} \leq 11 \times \psi^{-1 / 2} f_{t p}(w=-1.3 \psi)$,

where the RHS is a slowly decreasing function of the amplitude $\psi$ (see Eq. 33). Restricted to small amplitudes $\psi$, the condition can be simplified and expressed in physical units as $\left(\beta \lambda_{d}\right)^{-1}>0.5\left(e \psi / T_{e}\right)^{1 / 4}$. The inequality dictates that the scale length $\beta^{-1}$, or width of the electric spike, increases for a growing amplitude, a behaviour typical of electron holes (Muschietti et al., 1999a).

Second, the model imposes an ordering of the three time scales: $\omega_{\gamma} \ll \omega_{b} \ll \Omega_{e}$. Inequality Eq. (24) shows that the perpendicular width $\delta_{\perp}$ needs to increase for a decreasing ratio $\Omega_{e} / \omega_{e}$ lest the slow azimuthal drift becomes comparable to the bounce. It has been pointed out (Franz et al., 2000) that the solitary potential structures measured along magnetic field lines at various distances from Earth appear more oblate the smaller $\Omega_{e} / \omega_{e}$ is. Inequality Eq. (24) is consistent with this trend. We can also apply the inequality to the strongly magnetized potential structures measured by FAST. If we assume that the perpendicular scale $\delta_{\perp}$ is independently determined by a "channel" whose dimension is related to the ion gyroradius, we can invert Eq. (24) to obtain a condition on the parallel size $\Delta$. In dimensional units one finds

$\frac{\Delta}{\lambda_{d}} \ll \frac{-1}{\beta \lambda_{d}} \ln \left[\frac{2}{\left(\beta \lambda_{d}\right)^{2}}\left(\frac{\omega_{e}}{\Omega_{e}}\right)^{2}\left(\frac{\lambda_{d}}{\delta_{\perp}}\right)^{4} \frac{e \psi}{T_{e}}\right]$.

Substituting the numerical values of $\beta \lambda_{d}=1, \Omega_{e} / \omega_{e}=5$, $e \psi / T_{e}=1$, and $\delta_{\perp} / \lambda_{d}=10$ (for a $1 \mathrm{~km}$ channel), one obtains $\Delta \ll 12 \lambda_{d}$. Due to the logarithm, moderate changes to the parameter values do not significantly modify this inequality, and we conclude that the parallel size of a stretched solitary wave along $\boldsymbol{B}$ is limited to at most several Debye lengths.

The analysis presented says nothing about the stability of the nonlinear structure. In particular, the narrow lip bordering the "caldera" in Fig. 8 may seem a fragile element. We note, however, that the feature is very sensitive to the value of $\beta$. By decreasing $\beta$ from 1.3 to $\beta=0.8$ one can virtually eliminate it. Still, a powerful way to test the stability of the structure is to load it as an initial condition in a 3D particlein-cell code and explore its evolution or lack thereof within the parameter space. This would also be a good way to investigate the effects of the ions which have been assumed so far to provide a homogeneous background. In the frame of the solitary wave the ions appear as a cold beam impinging on the potential structure. Due to the lasting $E_{\perp}$ we can expect a significant transfer of momentum in the perpendicular direction, creating a wake behind the solitary wave. These questions cannot be addressed here and must await future work.

Acknowledgements. The research is supported by NASA grants NAG5-3596, NAG5-3916, and NAG5-8078 to the University of California.

\section{References}

Bender, C. M. and Orszag, S. A.: Advanced mathematical methods for scientists and engineers, McGraw-Hill, New York, 1978.

Bernstein, I. B., Greene, J. M., and Kruskal, M. D.: Exact nonlinear plasma oscillations, Phys. Rev., 108, 546-550, 1957.

Carlson, C. W., McFadden, J. P., Ergun, R. E., et al.: FAST observations in the downward auroral current region: Energetic upgoing electron beams, parallel potential drops and ion heating, Geophys. Res. Lett., 25, 2017-2020, 1998.

Cattell, C. A., Dombeck, J., Wygant, J. R., et al.: Comparisons of Polar satellite observations of solitary wave velocities in the plasma sheet boundary and the high altitude cusp to those in the auroral zone, Geophys. Res. Lett., 26, 425-428, 1999.

Ergun, R. E., Carlson, C. W., McFadden, J. P., et al.: FAST satellite observations of large-amplitude solitary structures, Geophys. Res. Lett., 25, 2041-2044, 1998.

Ergun, R. E., Carlson, C. W., Muschietti, L., et al.: Properties of fast solitary structures, Nonlinear Processes in Geophysics, 6, 187194, 1999.

Franz, J. R., Kintner, P. M., Seyler, C. E., et al.: On the perpendicular scale of electron phase-space holes, Geophys. Res. Lett., 27, 169-172, 2000. 
Goldman, M. V., Oppenheim, M. M., and Newman, D. L.: Nonlinear two-stream instabilities as an explanation for auroral bipolar wave structures, Geophys. Res. Lett., 26, 1821-1824, 1999.

Krasovsky, V. L., Matsumoto, H., and Omura, Y.: BernsteinGreene-Kruskal analysis of electrostatic solitary waves observed with Geotail, J. Geophys. Res., 102, 22, 131-139, 1997.

Mandrake, L., Pritchett, P. L., and Coroniti, F. V.: Electron beam generated solitary structures in a nonuniform plasma system, Geophys. Res. Lett., 27, 2869-2872, 2000.

Muschietti, L., Ergun, R. E., Roth, I., and Carlson, C. W.: Phasespace electron holes along magnetic field lines, Geophys. Res.
Lett., 26, 1093-1096; 1999a.

Muschietti, L., Roth, I., Ergun, R. E., and Carlson, C. W.: Analysis and simulations of BGK electron holes, Nonlinear Processes in Geophysics, 6, 211-219, 1999b.

Muschietti, L., Roth, I., Carlson, C. W., and Ergun, R. E.: A transverse instability of magnetized electron holes, Phys. Rev. Lett., 85, 94-97, 2000.

Tsurutani, B. T., Arballo, J. K., Lakhina, G. S., et al.: Plasma waves in the dayside polar cap boundary layer: Bipolar and monopolar electric pulses and whistler mode waves, Geophys. Res. Lett., 25, 4117-4120, 1998. 